\title{
Case Report \\ Pneumomediastinum as a Manifestation of Granulomatosis with Polyangiitis
}

\author{
Ahmed Alhazmi, ${ }^{1}$ Mohamed Moulaye Cheikh, ${ }^{2}$ and Rola Yousef Hassan (D) ${ }^{1}$ \\ ${ }^{1}$ Department of Medicine, Division of Rheumatology, King Fahad Armed Forces Hospital, Jeddah, Saudi Arabia \\ ${ }^{2}$ Doctor Soliman Fakeeh Hospital, Jeddah, Saudi Arabia \\ Correspondence should be addressed to Rola Yousef Hassan; r.toufiq@hotmail.com
}

Received 11 January 2020; Revised 28 June 2020; Accepted 8 July 2020; Published 31 July 2020

Academic Editor: Mehmet Soy

Copyright (C) 2020 Ahmed Alhazmi et al. This is an open access article distributed under the Creative Commons Attribution License, which permits unrestricted use, distribution, and reproduction in any medium, provided the original work is properly cited.

\begin{abstract}
A 38-year-old hypertensive male with a smoking history presented to the emergency room with significant hemoptysis, arthritis, and a purpuric rash. Other findings included a dropping hemoglobin and acute kidney injury with microscopic hematuria. The pulmonary computed tomography was significant for alveolar hemorrhage and a rarely reported pneumomediastinum. Along with this constellation of findings, a positive antiproteinase 3 helped to confirm this patient's diagnosis of granulomatosis with polyangiitis. Treatment commenced with sessions of plasma exchange and pulse steroids along with cyclophosphamide infusions. The patient had since improved and made a full recovery. This case report highlights the rarely described pneumomediastinum in association with vasculitides.
\end{abstract}

\section{Introduction}

Vasculitides are a group of uncommonly seen and diverse inflammatory diseases that affect blood vessels. These conditions may arise unprompted or may be precipitated by an underlying disease. It was first classified by the American College of Rheumatology in 1990 [1], with the Chapel Hill Consensus Conference providing a nomenclature system that more comprehensively covers all types of vasculitides, last updated in 2012 [2]. The most widely known branch of vasculitides is the antineutrophil cytoplasmic antibodypositive vasculitis (ANCA) which encompasses microscopic polyangiitis (MPA), granulomatosis with polyangiitis (GPA), and eosinophilic granulomatosis with polyangiitis (EGPA) [2].

GPA is a necrotizing, granulomatous vasculitis which affects multiple organs including the upper and lower respiratory tract and the kidneys [3]. Common pulmonary radiographic features include nodules, reticulations, segmental atelectasis, widespread opacities associated with air bronchograms, pleural lesions, and rarely hilar lymphadenopathy [4]. The most serious respiratory complication of this disease is alveolar hemorrhage, necessitating aggressive therapy with plasma-pharesis and immunosuppressive drugs [5].

Pneumomediastinum is the existence of air in the mediastinal cavity. This condition can either present spontaneously or secondary to manipulation of the patient's respiratory and digestive systems by trauma, infections, a perforated viscus, or via medical interventions [6]. Data reporting associations between pneumonediastinum and rheumatological diseases are limited, and this association, however, was more commonly pronounced in patients with concomitant interstitial lung disease, as with inflammatory myopathies, systemic lupus erythematosus and systemic sclerosis [7]. There was a case report published in 2014 documenting a presentation of pneumomediastinum in a patient with microscopic polyangitis that was preceded by interstitial pneumonia [8].

\section{The Case Report}

We are presenting the case of a 38-year-old male who was previously known to be hypertensive and a heavy smoker. $\mathrm{He}$ had presented to the emergency room with a 10-day 
history of productive cough which was associated initially with blood-tinged sputum but had escalated to massive hemoptysis. Further examination revealed the presence of arthritis and a purpuric rash scattered over his lower limbs (Figures 1 and 2). His arthritis was polyarticular, affecting mainly the small joints of the hands (the right first, second, and third proximal interphalangeal joints and the left second proximal interphalangeal joint were tender), and an ultrasound also confirmed grade 1 synovitis in both wrists.

The laboratory investigations were significant for a dropping hemoglobin, acute kidney injury, and microscopic hematuria (Table 1). On admission, his chest x-ray showed extensive bilateral ground glass opacities (Figure 3). A pulmonary highresolution computed tomography (HRCT) was ordered for further assessment and demonstrated evidence of alveolar hemorrhage in the form of diffuse scattered patchy areas of ground glass opacities in both lungs involving all lobes except the apical segments of the upper lung lobes and the subpleuretic areas (Figure 4). There was also a linear lucency noted in the left mediastinum representing pneumomediastinum (Figure 4). A preliminary diagnosis of pulmonary renal vasculitis was made, and a full autoimmune panel was sent.

A few hours after admission, the patient had developed significant hypoxia mandating transfer to the intensive care unit where he was stabilized and promptly started on pulse steroids (methylprednisone 1 gram intravenous daily) and plasma exchange sessions. He required intubation and mechanical ventilation two days after his admission due to deteriorating pulmonary functions and the development of acute respiratory distress syndrome. The patient was started urgently on cyclophosphamide $750 \mathrm{mg}$ intravascular infusion according to the National Institute of Health protocol. He underwent a skin biopsy which gave no evidence of vasculitis. A bronchoalveolar lavage was also performed and grew Pseudomonas and Alicyclobacillus for which he received colistin and tigecycline for a probable health-care-associated pneumonia (HCAP). After bronchoscopy, the patient had developed right pneumothorax which required the insertion of a chest tube.

The patient's overall condition gradually improved after receiving five sessions of plasma exchange and 5 days of pulse steroids. He was extubated and transferred to the medical ward. His test results came back positive for $\mathrm{C}$ antineutrophil cytoplasmic antibodies (cANCA) with a titer of $1: 20$ and anti-proteinase 3 (PR3), whilst P antineutrophil cytoplasmic antibodies (pANCA), myeloperoxidase (MPO), and the antiglomerular basement membrane (anti GBM) were negative (Table 1). A final diagnosis of granulomatosis with polyangitis was made after the exclusion of other causes. The differential included systemic lupus erythematosus which was ruled out based on the absence of supportive clinical features and autoantibodies (Table 1). Other differentials included Goodpasture's syndrome which was deemed less likely with negative anti-GBM and as this condition is not associated with arthritis or purpura. Other small vessel vasculitides such as eosinophilic granulomatosis with polyangitis and microscopic polyangitis were also less likely given that the patient gave no prior history of bronchial asthma, his investigation showed no eosinophilia, and a negative $\mathrm{P}$ ANCA/MPO (Table 1).

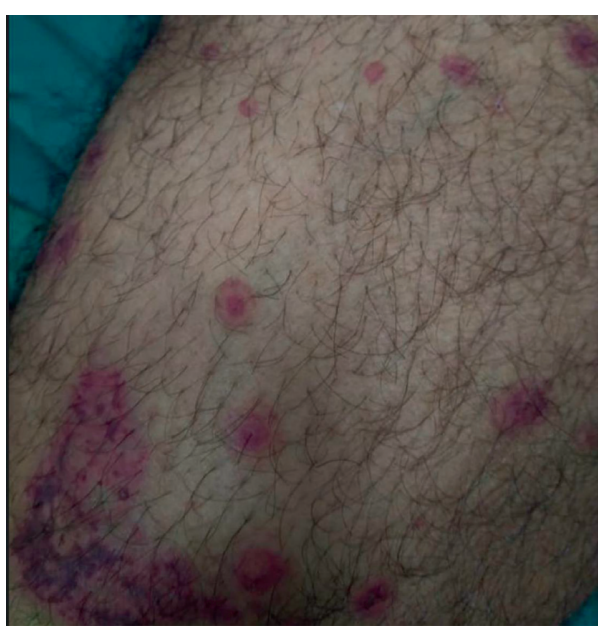

Figure 1: Purpuric rash scattered over the patient's lower limbs.

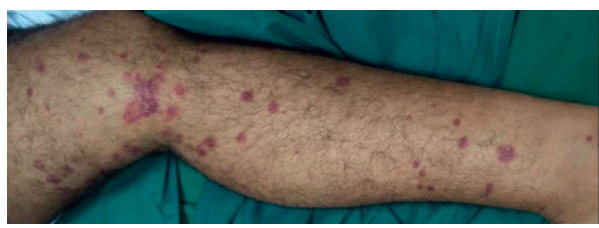

Figure 2: Purpuric rash scattered over the patient's lower limbs.

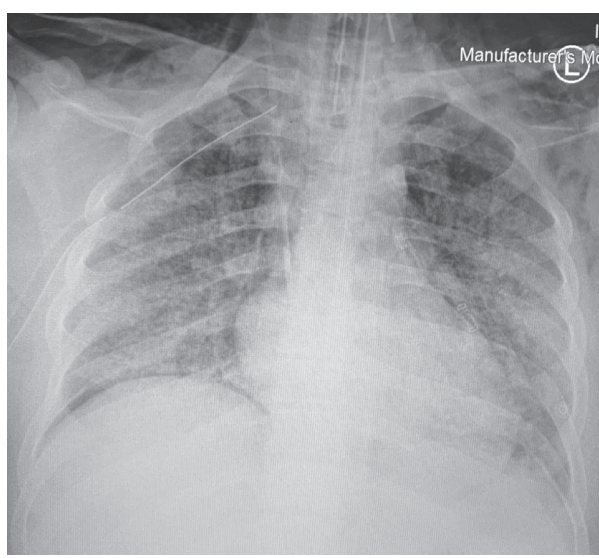

Figure 3: Admission chest X-ray showing bilateral extensive ground glass opacities.

The patient had received a second dose of cyclophosphamide during his stay. After a month spent in the hospital, the patient was discharged in a stable condition and given follow-up doses of cyclophosphamide along with tapering steroids in the outpatient setting. A repeated HRCT conducted 12 months after his initial presentation showed complete resolution of his ground glass opacities and pneumomediastinum (Figure 5).

\section{Discussion}

GPA is a multisystemic disease with involvement mainly affecting the respiratory and renal systems [3]. One of the earliest and most impactful papers that closely studied the 
TABLE 1: Laboratory investigations.

\begin{tabular}{|c|c|c|}
\hline & Pretreatment & 3 months after treatment \\
\hline White blood cells & $10.9 \times 109$ & $8.67 \times 109$ \\
\hline Absolute neutrophil count & $5.6 \times 109$ & $8.1 \times 109$ \\
\hline Absolute lymphocyte count & $1.9 \times 109 / \mathrm{L}$ & $3.9 \times 109 / \mathrm{L}$ \\
\hline Absolute eosinophil count & $0.2 \times 109 / \mathrm{L}$ & $0.108 \times 109 / \mathrm{L}$ \\
\hline Hemoglobin & $8.68 \mathrm{~g} / \mathrm{dL}$ & $12.9 \mathrm{~g} / \mathrm{dL}$ \\
\hline Platelet & $265 \times 109$ & $334 \times 109$ \\
\hline Creatinine & $120 \mathrm{umol} / \mathrm{L}$ & $114 \mathrm{umol} / \mathrm{L}$ \\
\hline Urea & $14 \mathrm{nmol} / \mathrm{L}$ & $14 \mathrm{umol} / \mathrm{L}$ \\
\hline C-reactive protein & $6.5 \mathrm{mg} / \mathrm{L}$ & $5.5 \mathrm{mg} / \mathrm{L}$ \\
\hline Erythrocyte sedimentation rate & $110 \mathrm{MM} / \mathrm{HR}$ & $17 \mathrm{MM} / \mathrm{HR}$ \\
\hline \multicolumn{3}{|l|}{ Urine analysis } \\
\hline Blood & +3 & +1 \\
\hline Red blood cells & $+2(10-34$ cells $)$ & Negative \\
\hline Protein & Negative & Negative \\
\hline White blood cells & Negative & Negative \\
\hline Casts & No casts seen & No casts seen \\
\hline Antinuclear $\mathrm{Ab}$ & $<1: 40$ & \\
\hline P ANCA & $<1: 20$ & $<1: 20$ \\
\hline C ANCA (indirect immunofluorescence assay) & $1: 20$ & $<1: 20$ \\
\hline PR3 & $26.2 \mathrm{kU} / \mathrm{L}$ & \\
\hline MPO & $1.4 \mathrm{kU} / \mathrm{L}$ & \\
\hline Anti-GBM Ab & $0.9 \mathrm{U} / \mathrm{mL}$ & \\
\hline
\end{tabular}

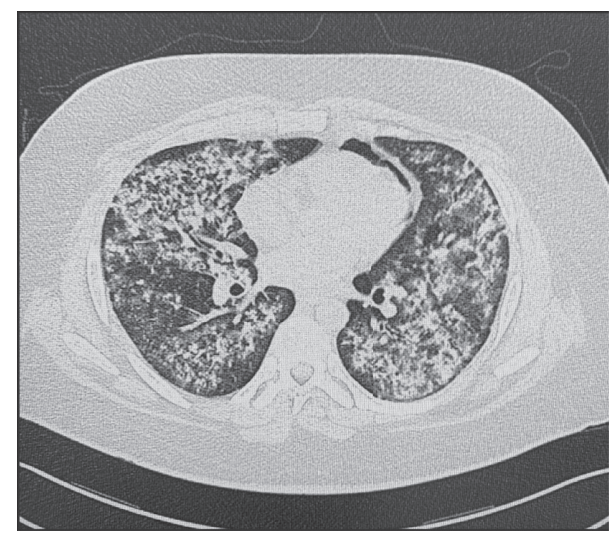

FIgURE 4: Pretreatment high resolution computed tomography.

pattern of GPA lung involvement is the article by Cordier et al. [4]. The authors had characterized the patterns and frequencies of pulmonary findings in 77 patients with biopsy proven GPA. Of this population of patients, 53 " $69 \%$ " had presented with nodular manifestations, while the occurrence of infiltrates was found in 41 patients " $53 \%$." The incidence of pleural effusions was established in 9 patients " $12 \%$," and only 3 patients " $4 \%$ " had developed atelectasis. Furthermore, alveolar hemorrhage as a presenting symptom was only reported in 6 patients $(8 \%)$, and in contrast to our case report findings, pneumomediastinum had not developed in any of these cases.

Pneumomediastinum is the presence of air in the mediastinum. This phenomenon may occur either due to an underlying precipitant or even with the lack thereof. Trauma, infections, and procedures are all known culprits [6]. At the time of our patient's presentation, he had none of these well-described secondary causes. Caceres et al. [6] had further described a different set of factors that could

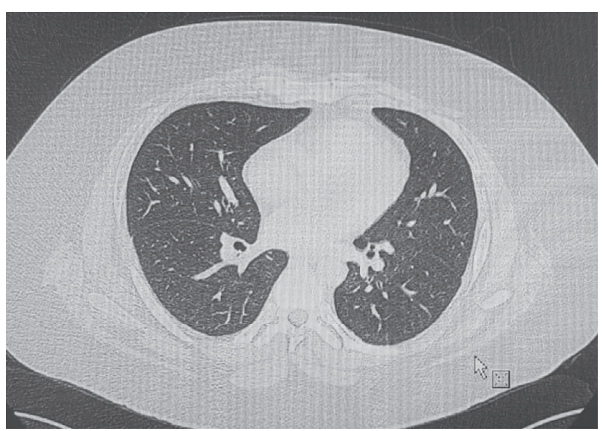

Figure 5: Posttreatment high resolution computed tomography.

predispose to the development of spontaneous pneumomediastinum. These factors include smoking, inhaled drugs, and airway lung disease such as asthma and chronic obstructive pulmonary disease, as well as idiopathic pulmonary fibrosis. Although smoking was present in our case and might have been a contributing factor to the patient's development of pneumomediastinum, a direct causality relationship cannot be substantiated as the patient had also presented with an active vasculitic lung pathology.

Associations between pneumomediastinum and autoimmune diseases have been scarcely mentioned in the literature. These associations were mainly seen in conditions with a predilection for the development of interstitial lung disease. There have been a few case reports linking pneumomediastinum with systemic sclerosis, systemic lupus erythematosus, and rheumatoid arthritis [9-11]. Pneumomediastinum was also found to be predictive of poor prognosis in patients with dermatomyositis [12]. However, regarding associations with ANCA vasculitis, the only report found was in a patient with a preexisting interstitial pneumonia who developed microscopic polyangitis [8]. 
Histopathological features of vasculitic pulmonary affection had been delineated by Homma et al. [13] who had studied 31 patients with myeloperoxidase ANCA-associated vasculitis and pulmonary fibrosis. Findings included vasculitic changes in pulmonary arteries and arterioles in three cases each and in capillaries presenting with alveolar hemorrhages in two cases. The characteristic histological features of vasculitis found in pulmonary arterioles were leukocytoclastic angiitis, intimal elastofibrosis with destroyed elastic fibers, and granulomatous angiitis, each in one of the three cases mentioned. Proposed underlying mechanisms for pneumomediastinum on the other hand may include mucosal necrosis in the airways as a result of vasculitic processes at the level of bronchial arterioles, a process that may also manifest with cutaneous vasculopathy, suggest Kono et al. who had studied patients with dermatomyositis and pneumomediastinum [14]. Based on previously laid data, we can extrapolate the pathological pathway of our case, which may have started with mucosal necrosis secondary to GPA vasculitis and subsequent rupture of vulnerable alveolar connective tissue leading to the development of pneumomediastinum.

We hope to further enrich the medical literature with our addition of the first documented association of GPA and pneumomediastinum and to pave the way for more research in this field.

\section{Data Availability}

Access to data can be carried out through hospital records.

\section{Ethical Approval}

Ethical approval from the hospital's Research and Ethics Committee was obtained.

\section{Consent}

Informed consent from the patients for publication of their data was obtained.

\section{Conflicts of Interest}

The authors declare that they have no conflicts of interest.

\section{References}

[1] D. A. Bloch, B. A. Michel, G. G. Hunder et al., "The American college of rheumatology 1990 criteria for the classification of vasculitis: patients and methods," Arthritis \& Rheumatism, vol. 33, pp. 1068-1073, 1990.

[2] J. C. Jennette, R. J. Falk, P. A. Bacon et al., "2012 revised international Chapel Hill Consensus conference nomenclature of vasculitides," Arthritis \& Rheumatism, vol. 65, no. 1, pp. 1-11, 2013.

[3] R. J. Falk, W. L. Gross, L. Guillevin et al., "Granulomatosis with polyangiitis (Wegener's): an alternative name for Wegener's granulomatosis," Arthritis \& Rheumatism, vol. 63, no. 4 , pp. $863-864,2011$, p.
[4] J.-F. Cordier, D. Valeyre, L. Guillevin, R. Loire, and J.-M. Brechot, "Pulmonary Wegener's granulomatosis," Chest, vol. 97, no. 4, pp. 906-912, 1990.

[5] M. Yates, R. A. Watts, I. M. Bajema et al., "EULAR/ERAEDTA recommendations for the management of ANCA-associated vasculitis," Annals of the Rheumatic Diseases, vol. 75, no. 9, pp. 1583-1594, 2016.

[6] M. Caceres, S. Z. Ali, R. Braud, D. Weiman, and H. E. Garrett, "Spontaneous pneumomediastinum: a comparative study and review of the literature," The Annals of Thoracic Surgery, vol. 86, no. 3, pp. 962-966, 2008.

[7] E. J. Dein, K. Lee, H. Timlin, and L. Hummers, "Spontaneous pneumomediastinum in limited cutaneous systemic sclerosis and myositis overlap," BMJ Case Reports, vol. 2018, pp. bcr2018, 2018.

[8] K. Isoda, T. Takeuchi, T. Ishida, S. Makino, and T. Hanafusa, "Pneumomediastinum in a patient with microscopic polyangiitis preceded by interstitial pneumonia," Internal Medicine, vol. 53, no. 8, pp. 891-893, 2014.

[9] M. Haroon, P. McLaughlin, M. Henry, and S. Harney, "Spontaneous pneumomediastinum in a patient with anticentromere antibody-positive limited scleroderma," Journal of Clinical Rheumatology, vol. 17, no. 1, pp. 42-43, 2011.

[10] S. O. Paria and S. Roverano, "Bilateral pneumothorax and mediastinal emphysema in systemic lupus erythematosus," Clinical Rheumatology, vol. 11, no. 4, pp. 571-573, 1992.

[11] A. Patel, B. Kesler, and R. A. Wise, "Persistent pneumomediastinum in interstitial fibrosis associated with rheumatoid arthritis," Chest, vol. 117, no. 6, pp. 1809-1813, 2000.

[12] B. Le Goff, P. Chérin, A. Cantagrel et al., "Pneumomediastinum in interstitial lung disease associated with dermatomyositis and polymyositis," Arthritis Care \& Research, vol. 61, no. 1, pp. 108-118, 2008.

[13] S. Homma, H. Matsushita, and K. Nakata, "Pulmonary fibrosis in myeloperoxidase antineutrophil cytoplasmic antibody-associated vasculitides," Respirology, vol. 9, no. 2, pp. 190-196, 2004.

[14] H. Kono, S. Inokuma, H. Nakayama, and M. Suzuki, "Pneumomediastinum in dermatomyositis: association with cutaneous vasculopathy," Annals of the Rheumatic Diseases, vol. 59 , no. 5 , pp. $372-376,2000$. 